\title{
DEFENSA DEL RECONOCIMIENTO DE UN DERECHO CONSTITUCIONAL DEL MEDIO AMBIENTE A NIVEL EUROPEO
}

\author{
DEFENSE OF THE RECOGNITION OF A \\ CONSTITUTIONAL RIGHT OF THE ENVIRONMENT \\ AT THE EUROPEAN LEVEL
}

\begin{tabular}{|c|c|}
\hline \multicolumn{2}{|c|}{ ARTÍCULO INÉDITO DE INVESTIGACIÓN } \\
\hline CÓMO CITAR ESTE ARTÍCULO (CHICAGO) & $\begin{array}{l}\text { De Sadeleer, Nicolas. "Defensa del } \\
\text { reconocimiento de un derecho } \\
\text { constitucional del medio ambiente } \\
\text { a nivel europeo". Revista de Derecho } \\
\text { Aplicado LLM UC } 6 \text { (2020). } \\
\text { doi: Io.7764/rda.o.6.16693. }\end{array}$ \\
\hline REVISTA DE DERECHO APLICADO LLM UC & $\begin{array}{l}\text { Número } 6 \\
\text { Diciembre } 2020 \\
\text { ISSN: } 245^{2-4344}\end{array}$ \\
\hline & $\begin{array}{l}\text { Recepción: I de julio, } 2020 \\
\text { Aceptación: } \text { I }_{4} \text { de septiembre, } 2020\end{array}$ \\
\hline
\end{tabular}




\section{Resumen}

Aunque durante la década de los ochenta ya existía una amplia legislación secundaria que abarcaba el agua y el aire, el ruido, los productos químicos, los desechos y la protección de la naturaleza, no había competencias ambientales específicas en el tratado. Con el objetivo de llenar este vacío, el Acta Única Europea, así como los Tratados de Maastricht, Ámsterdam, Niza y Lisboa, han ido perfeccionando progresivamente una competencia medioambiental de la Unión Europea más sólida, que persigue objetivos ambiciosos y se basa en una serie de principios. Junto con el reconocimiento del desarrollo sostenible, las obligaciones medioambientales del derecho primario instan a las instituciones de la Unión Europea y a los 27 Estados miembros a desglosar sus diferentes políticas de acuerdo con las consideraciones medioambientales. Si bien puede que no constituyan una revolución, estos sucesivos ajustes del Tratado son un testimonio de la constitucionalización de la política medioambiental a nivel de la Unión Europea.

Palabras clave: Desarrollo sostenible, integración de preocupaciones ambientales, alto nivel de protección ambiental, principios ambientales, derecho a la protección ambiental.

\section{Abstract}

Though there was already extensive secondary legislation covering water and air, noise, chemicals, waste, and nature protection during the $80 \mathrm{~s}$, there were no specific environmental competencies in the treaty. Aiming at filling this gap, the Single European Act, the Treaties of Maastricht, Amsterdam, Nice, and Lisbon have been progressively honing a more robust EU environmental competence that pursues ambitious objectives and is reckoning upon a range of principles. Alongside the recognition of sustainable development, the primary law environmental obligations call the EU institutions and the 27 Member States to decompartmentalize their different policies in line with environmental considerations. Whilst they may not amount to a revolution, these successive Treaty adjustments are a testament to the constitutionalization of the environmental policy at the EU level.

Keywords: Sustainable development, integration of environmental concerns, high level of environmental protection, environmental principles, right to environmental protection. 


\section{Nicolas de Sadeleer}

Université Saint-Louis

Bruselas, Bélgica

nicolas.desadeleer@usaintlouis.be

Université Saint-Louis

Brussels, Belgium

nicolas.desadeleer@usaintlouis.be
Profesor de la Université Saint-Louis, Bélgica (Chaire Jean Monnet). Es especialista en derecho de la Unión Europea (instituciones, mercado interior), derecho ambiental (internacional y nacional) y derecho comparado. Es un activo comentarista sobre cuestiones legales y políticas de la Unión Europea en las áreas de comercio, inversión y desarrollo sostenible. Es doctor en Derecho por la Université Saint-Louis. Su investigación ha sido publicada por las principales editoriales y revistas académicas en el mundo.

Professor at Université Saint-Louis, Belgium (Chaire Jean Monnet). He is a specialist in European Union law (institutions, internal market), environmental law (international and domestic), and comparative law. He is an active commentator on European Union legal and political issues in the areas of trade, investment, and sustainable development. Doctor of Law, Université Saint-Louis. His research has been published by leading scholarly publishing houses and journals around the globe. 


\section{INTRODUCCIÓN}

Aunque la protección del medio ambiente no es una preocupación reciente, ha adquirido una nueva urgencia en los últimos años, marcada por la necesidad apremiante de encontrar soluciones universales al calentamiento de global, la erosión de la diversidad biológica y el agotamiento de los recursos naturales. De hecho, no pasa un día sin que se nos informe de las nuevas amenazas, riesgos insospechados, derrames de petróleo, desaparición de especies silvestres, incendios devastadores, derretimiento de los glaciares, niebla tóxica troposférica, interruptores endocrinos... Desde su marginalidad, este tema se ha convertido rápidamente en una cuestión importante. El interés se debe sin duda al hecho de que la situación se ha vuelto alarmante y es probable que empeore si no se emprende nada ambicioso.

¿Qué pasa con el derecho de la Unión Europea en todo esto? En primer lugar, al estar compuesta por veintisiete Estados - que representan una duodécima parte de la población mundial-, esta organización ha garantizado un desarrollo económico sin precedentes, cuyo impacto en el medio ambiente tanto continental como mundial está lejos de ser insignificante. Las políticas en materia de pesca, mercado interior, desarrollo regional, turismo, transporte y comercio exterior fomentan, de un modo u otro, la explotación o incluso la sobreexplotación de los recursos naturales. Francamente, este modelo económico no está exento de crítica y el Acuerdo Verde, que fue lanzado en $2019^{1}$, tiene como objetivo corregir algunas de sus deficiencias.

En segundo lugar, los sistemas jurídicos son ambivalentes en cuanto a que las normas que segregan pueden tanto agravar los riesgos ambientales como contenerlos. Este es sin duda el caso del ordenamiento jurídico de la Unión. Teniendo sus orígenes en una lógica de integración económica que ignoraba la escasez de recursos naturales, este orden se ha abierto lentamente a las preocupaciones medioambientales ${ }^{2}$. Aunque no se mencionaba en 1957 en el Tratado de Roma, este hecho ha logrado, en el curso de las reformas institucionales, imponerse como una de las preocupaciones esenciales de las instituciones. Inspirada a veces por el temor a un mercado común que se desmorona, a veces por el deseo de mostrar una imagen menos mercantil, a veces por el deseo de salvaguardar la naturaleza amenazada, nació así una política europea hace casi cuatro décadas. Esta política y la ley que ha generado han logrado imponerse tanto a los actores públicos como a los privados. Hoy en día existen más de 300 leyes reglamentarias, es decir, casi el 8\% de la

1 “El Pacto Verde Europeo”, Comisión Europea, Ir de diciembre de 2org, https://eur-lex.europa. $\mathrm{eu} /$ legal-content/ES/TXT/2qid=I588580774010\&uri=CELEX\%3 ${ }_{3}{ }_{5}^{2019 D C o 6}{ }_{4} \mathrm{O}$.

2 Nicolas de Sadeleer, Commentaire Mégret Environnement et marché intérieur (Bruselas: Éditions de l'Université libre de Bruxelles, 20I0). 
legislación secundaria, cuya aplicación da lugar a una gran cantidad de controversias. La legislación secundaria regula el impacto y la contaminación (ruido, residuos, sustancias químicas, emisiones de agua y aire) y protege los principales ecosistemas (agua, aire), así como algunos de sus componentes (fauna y flora).

La importancia actual del derecho ambiental dentro de la legislación secundaria no debe ocultar importantes debilidades. El carácter complejo e inestable de estos fenómenos, que estas normas jurídicas pretenden contrarrestar, obliga al legislador a poner en forma constante su esfuerzo sobre la mesa. La ley resultante es particularmente densa e incomprensible; se pierde en una profusión de reglas. Abandonando toda pretensión de regular lo esencial, parece condenada a apartarse de los imperativos de generalidad y abstracción que han conformado el derecho moderno. Tanto su enredo como la naturaleza técnica de estas regulaciones son suficientes para hacer retroceder incluso a los juristas más temerarios. Por lo tanto, se trata más de un enredo reglamentario que de un sistema bien definido.

Por otra parte, a pesar de los esfuerzos, los resultados del derecho ambiental y de la política del mismo nombre siguen siendo mixtos. No obstante el éxito de ciertas medidas, no se han alcanzado los objetivos que persiguen muchas reglamentaciones - por ejemplo, la reducción de la contaminación industrial-, sin duda debido a los fenómenos de acumulación resultantes del consumo masivo, al parecer ilimitado. Por consiguiente, cualquier progreso alcanzado en materia de reglamentación se ve rápidamente superado por la aparición de nuevos fenómenos, o incluso de riesgos insospechados, como la biotecnología o la nanotecnología.

¿Significa esto que debemos perdernos en los resquicios de los reglamentos técnicos? ¿O deberíamos, por el contrario, aclarar los fundamentos constitucionales para una verdadera protección del medio ambiente en las normas del derecho primario? Mientras que el derecho secundario está destinado a desaparecer debido a la constante adaptación al progreso técnico y científico, el derecho primario está aquí para quedarse.

El derecho primario es un verdadero laboratorio de ideas, lleno de disposiciones que reflejan la búsqueda de la excelencia en la protección del medio ambiente, que analizaremos brevemente en esta publicación. Además, la protección del medio ambiente siempre se ha caracterizado por su propensión a exportar sus principios y conceptos. Las obligaciones de integración, un alto nivel de protección y las medidas de precaución, que se introdujeron en primer lugar como parte de la política ambiental, se extendieron rápidamente a otras esferas jurídicas.

Destacaremos aquí las principales obligaciones constitucionales que se encuentran en los tratados fundacionales, que podrían servir de base para el derecho constitucional ambiental europeo. Como cualquier otra disposición de carácter constitucional relativa a la política pública, estas obligaciones también tienen muchas debilidades que destacamos aquí. 


\section{DESARROLLO DURADERO}

Durante los tres primeros decenios de su existencia, el reto inicial del derecho ambiental fue controlar los impactos y contaminación de origen industrial mediante regímenes administrativos. Esto explica el lugar eminente que ocupa el derecho de los establecimientos clasificados y la regulación del impacto industrial en esta rama del derecho.

Hoy en día, se puede criticar a los iniciadores de esta política por haber dejado de lado las cuestiones relacionadas, por un lado, con la extracción de recursos naturales - cuya explotación parecía ilimitada - y, por otro lado, con un consumo cada vez mayor de bienes y servicios. El historial de la política ambiental sigue siendo mixto precisamente por esta incapacidad de regular, antes, la explotación de los recursos naturales y, después, el consumo desenfrenado. No tiene sentido equipar los vehículos de motor con nuevas tecnologías si el número de automóviles y los kilómetros recorridos aumentan en forma constante ("efecto rebote"). ¿Qué sentido tiene someter el transporte aéreo a un sistema de cuotas de emisiones de gases de efecto invernadero si estas siguen aumentando? ¿Cómo se puede desarrollar una política de productos sustentables si los consumidores compran un nuevo teléfono móvil en promedio cada catorce meses?

Bajo el impulso del desarrollo sustentable, el derecho ambiental debería intervenir en mayor medida tanto antes como después. En primer lugar, antes: dado que la explotación de los recursos naturales no es infinita, no se debe seguir abusando de ellos; deben ser usados sabiamente. También después, ya que el consumo desenfrenado conduce a la sobreexplotación de los recursos naturales y a una gran cantidad de impactos negativos.

En contraste con las referencias dispersas e imprecisas al desarrollo sustentable en los tratados anteriores, el nuevo párrafo 3 del artículo 3 del Tratado de la Unión Europea expresa más claramente la naturaleza triple del principio. Así pues, el "desarrollo sustentable de Europa" se presenta como "basado" sucesivamente en preocupaciones económicas ("crecimiento económico equilibrado"), sociales ("una economía social de mercado [...] con miras al pleno empleo y el progreso social") y ambientales ("un alto nivel de protección y mejora de la calidad del medio ambiente"). Además, "en sus relaciones con el resto del mundo", la Unión contribuirá, en particular, "al desarrollo sustentable del planeta" (artículo 3.5 del Tratado). Por otra parte, el desarrollo sustentable se menciona tanto en el artículo 11 del Tratado de Funcionamiento de la Unión Europea (cláusula de integración) como en el artículo 37 de la Carta (derecho de medio ambiente). Esta multiplicación de referencias al concepto de desarrollo sustentable no debe ser engañosa. A los ojos de la doctrina, este concepto se asemeja más a un objetivo político que a un principio jurídico.

Aunque estas diversas disposiciones no son vinculantes, no obstante, establecen un imperativo político: el "alto nivel de protección y mejora de la calidad del medio ambiente" se sitúa ahora al mismo nivel que el objetivo, por ejemplo, "del crecimiento económico" 
(pilar económico) y "una economía social de mercado altamente competitiva” (pilar social del desarrollo sustentable). Como no se ha establecido una jerarquía entre estas diferentes misiones, forman un todo indisociable y, por lo tanto, no pueden ser interpretadas de forma aislada. Por consiguiente, el principal objetivo de promover el progreso económico y social dentro de la Unión debe considerarse en una perspectiva equilibrada y duradera.

\section{LA BÚSQUEDA DE UN ALTO NIVEL DE PROTECCIÓN DEL MEDIO AMBIENTE}

Según el párrafo 3 del artículo 3 del Tratado, la obligación de lograr "un alto nivel de protección y mejora de la calidad del medio ambiente" es una de las tareas asignadas a la Unión Europea. A riesgo de repetirse, los autores establecieron una obligación idéntica en el contexto del procedimiento de adopción de legislación secundaria, que persigue la protección del medio ambiente como objetivo primario (artículo 191, numeral 2 del Tratado de Funcionamiento) o secundario (artículo 114, numeral 3). Esto no es exclusivo de las cuestiones ambientales: las políticas de salud pública y de protección del consumidor recogen este requisito cualitativo (artículo 168, párr. 1 y artículo 169, párr. 1).

Dado que ya no se trata solo de una cuestión de protección, sino también de "mejorar la calidad del medio ambiente", esta obligación es de naturaleza dinámica. Se trata de una acción transformadora y no conservadora que, por lo tanto, se espera de las instituciones. Las instituciones no solo están obligadas a prevenir la degradación, sino también a tratar activamente de mejorar la calidad del medio ambiente y la calidad de vida de los ciudadanos. En esta etapa, nos gustaría hacer dos observaciones.

La primera observación es que, al evocar ese objetivo cualitativo, el derecho primario pone la vara mucho más alta que otros tratados internacionales. Estos acuerdos están más en línea con una perspectiva reactiva que proactiva. De hecho, en general se exige a las partes que no causen "daños importantes" ${ }^{\text {ni }}$ "cambios climáticos antropógenos peligrosos" ${ }^{4}$

La segunda observación, ya sea para la política de mercado interno o la política ambiental, es que la redacción de esta obligación es desconcertante. De hecho, el umbral puede ser objeto de diversas evaluaciones. Una medida propuesta por la Comisión puede parecer draconiana a los ojos de los Estados cuya política es laxa, pero insuficiente para otros

3 Artículo 7, párr. I, de la Convención sobre el derecho de los usos de los cursos de agua internacionales para fines distintos de la navegación (Nueva York, 2i de mayo de I997, aún no en vigor).

4 Convención sobre el Cambio Climático (Nueva York, 9 de mayo de I992, entrada en vigor el 2I de marzo de 1994). 
Estados. 2 Debe darse prioridad a la protección máxima? ¿Debería lograrse el "riesgo cero" ${ }^{5}$ ? 2 Se puede deducir de estas disposiciones que el nivel de protección sería el más alto posible? ¿ $\mathrm{O}$ solo se debe proporcionar una protección intermedia? Excepcionalmente, ¿podría lograrse la armonización sobre la base del mínimo común denominador? La indeterminación de esta obligación no significa, sin embargo, que las instituciones gocen de una discreción absoluta al respecto.

\section{INTEGRACIÓN DE LOS REQUISITOS AMBIENTALES}

La protección del medio ambiente ha cedido en la mayoría de los casos a los imperativos socioeconómicos. Así pues, en los concursos de política administrativa, las soluciones jurisprudenciales adoptadas tienden por lo general a favorecer el desarrollo económico y no la conservación de los recursos naturales. Como consecuencia, la naturaleza ha pagado un alto precio por la falta de integración de los requisitos ambientales en otras políticas.

En el derecho de la Unión Europea, el derecho ambiental interactúa constantemente con otras ramas del derecho. Además de la aproximación de las legislaciones con miras a facilitar la realización del mercado interior (artículos 114 a 118 del Tratado de Funcionamiento), la política medioambiental interactúa a cada momento con la política de transportes (título 6), política comercial común (parte 5, título 2), salud pública (título 14), protección de los consumidores (título 15), redes transeuropeas (título 16), industria (título 17), cohesión económica y social (título 18) y cooperación para el desarrollo (1uinta parte, título 3, capítulo 1, artículo 5.1). Así pues, aunque el Tratado de Funcionamiento sigue insistiendo en una "economía de mercado abierta con libre competencia" (artículo 119, número 1), ahora es preciso armonizar todas las políticas, en particular con miras a promover el desarrollo sustentable (artículo 11).

Por lo tanto, era esencial, más allá del reconocimiento del desarrollo sustentable, descomponer las diversas políticas de la Unión, con el fin de integrar mejor las consideraciones ambientales en ellas.

El deber de integración ocupa ahora un lugar simbólico entre las disposiciones introductorias del Tratado, en el título 2, sobre disposiciones de aplicación general. Así pues, el artículo 11 del Tratado de Funcionamiento dispone que "las exigencias de la protección del medio ambiente deberán integrarse en la definición y en la realización de las políticas y acciones de la Comunidad a que se refiere el artículo 3, en particular con objeto de fomen-

5 La obligación de lograr un alto nivel de protección de la salud (artículo i68 del Tratado de Funcionamiento; artículo I52 del Tratado) lleva a veces al legislador de la Unión a mostrar una "tolerancia cero" en el ámbito de la seguridad alimentaria (Corte de Justicia, I de abril de 2004, Bellio Flli, C-286/o2, Rec., p. I-3465). 
tar un desarrollo sustentable". Este requisito contribuye al reconocimiento de un valor trascendental ${ }^{6}$. Esta cláusula debería desempeñar un papel importante, no solo porque evita interferencias y contradicciones entre políticas en competencia, sino también porque promueve la elaboración de políticas globales, coherentes y eficaces. Debe permitir que la política ambiental vaya más allá del alcance limitado que generalmente se le reserva.

Sin embargo, la obligación de integración no es exclusiva de la política ambiental. Forma parte de un objetivo general de coherencia entre las diferentes políticas y acciones de la Unión (artículo 7 del Tratado de Funcionamiento). Además, otras disposiciones del Tratado de Funcionamiento, ya sea en el ámbito de la salud o de la producción de consumo, proclaman el carácter transversal de los intereses legítimos de los ciudadanos de la Unión?

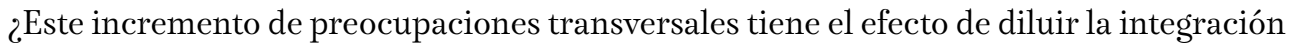
de los requisitos ambientales como sustituto de buenas intenciones? ¿Qué peso podemos dar ahora al medio ambiente en relación con la cultura o la industria? ¿Cómo se deben priorizar estos valores?

Sin embargo, a pesar de la indeterminación de sus normas de aplicación, cabe señalar que el artículo 11 del Tratado de Funcionamiento es de carácter vinculante (“deberá") y que su ámbito de aplicación material es particularmente amplio ("definición y ejecución de las políticas y actividades de la Unión”). Por lo tanto, la cláusula no solo obliga a las instituciones a intervenir, sino que también las lleva a ampliar el alcance de su intervención. De hecho, los términos "políticas y acciones" abarcan todas las medidas, incentivos y planes, no se limitan a los elementos denominados "políticas" y "acciones".

Sin embargo, una de sus principales debilidades radica en que los instrumentos conceptuales para su aplicación no han sido definidos por los autores de los tratados. Los principios ambientales del artículo 191 del Tratado de Funcionamiento podrían ser los

6 Martin Wasmeier, "The Integration of Environmental Protection as General Rule for Interpretating Law", Common Market Law Review 38, n. ${ }^{\circ}$ I (200I): I59-I77; Carlos-Manuel Alves, "La protection intégrée de l'environnement en droit de l'UE" (tesis de la Universidad de Montesquieu-Burdeos IV, 2002), 9०3; Nele D'Hondt, Integration of Environmental Protection into other European Community Policies: Legal Theory and Practice (Groninga: Europa Law Publishing, $2003), 643$.

7 Se trata de la igualdad entre hombres y mujeres (artículo 8 del Tratado de Funcionamiento), la promoción de un alto nivel de empleo (artículo 9), la lucha contra la discriminación (artículo Io), la protección de los consumidores (artículo i2), la cultura (artículo i67, apartado 4 del Tratado de Funcionamiento; artículo I5I, párr. 4 del Tratado de la Comunidad Europea), la salud (artículo i68, párr. I del Tratado de Funcionamiento), la industria (artículo I73, párr. 3) y la cooperación para el desarrollo (artículo 208, párrs. I-2). 
brazos armados de la obligación de integración, pero el juez de la Unión parece reacio a seguir este camino ${ }^{8}$. De igual modo, el grado de integración requerido no se especifica en el Tratado y, en la práctica, a veces varía considerablemente de una política a otra.

Otra dificultad es que el propósito de la integración es el "desarrollo sustentable" (artículo 11 del Tratado de Funcionamiento) o "el principio del desarrollo sustentable" (artículo 37 de la Carta). Sin embargo, en la medida en que el desarrollo sustentable tiende precisamente, como ya hemos subrayado, a conciliar los intereses económicos, sociales y medioambientales, las exigencias medioambientales corren el riesgo de ser dejadas de lado en nombre de imperativos contradictorios. El resultado es cuando menos paradójico: mientras que el artículo 11 del Tratado de Funcionamiento parece dar prioridad a la protección del medio ambiente, la promoción del desarrollo sustentable podría, por el contrario, diluir o incluso anular el alcance de la cláusula de integración.

Por último, el Tribunal de Justicia recurre regularmente al artículo 11 del Tratado de Funcionamiento cuando debe pronunciarse sobre casos difíciles ${ }^{9}$; la cláusula de integración adquiere entonces los rasgos de un principio de interpretación.

\section{PRINCIPIOS}

En contraste con un sistema cerrado que evoluciona en completa autonomía, el derecho de la Unión se encuentra constantemente en la interfaz del derecho nacional y regional y el derecho internacional. Al armonizar las reglamentaciones nacionales, el legislador de la Unión extrae de ellas su contenido sustancial y establece así los principios que le guiarán en su tarea. A la inversa, incorpora en su ordenamiento jurídico los principios establecidos en las convenciones internacionales que celebra.

El título 20 del Tratado de Funcionamiento (artículos 191 a 193), dedicado exclusivamente a la política de medio ambiente, no se limita a confirmar la competencia de la Unión en materia de medio ambiente: junto a los objetivos enunciados, hay una serie de principios que exigen, por tanto, una acción de la Unión más fuerte y coherente.

8 El Tribunal subrayó que el principio de precaución "es el corolario, entre otras cosas, del principio de la primacía de los requisitos de protección de la salud sobre los intereses económicos" (TPI, 26 noviembre 2002, Artegodan, T-74/00, Rec., p. II-4945, punto I74).

9 Corte de Justicia de la Unión Europea, io de enero de 2006, Comisión con Consejo, C-94/o3, [2006] ECR I-I, párrafo 26; conclusiones del abogado general Jacobs en el marco de la Corte, I3 de marzo de 200I, PreussenElektra, C-379/98, ECR, p. I-2I59, punto 232; Corte de Justicia de la Unión Europea, I5 de noviembre de 2005, Comisión con Austria, C-320/03, [2005] ECR I-987I, punto 73; Corte de Justicia de la Unión Europea, 13 de septiembre de 2005, Comisión con Consejo, C-I76/03, [2005] ECR I-7879, punto $4_{4}^{2}$. 
El artículo 191(2) del Tratado de Funcionamiento lo establece:

La política [...] sobre el medio ambiente [...] se basará en el principio de precaución y en los principios de que se deben adoptar medidas preventivas, de que el daño ambiental debe rectificarse prioritariamente en la fuente y de que quien contamina debe pagar.

Así, pues, a diferencia de las demás políticas de la Unión, la política de medio ambiente proclama principios que se derivan tanto del orden jurídico internacional como del orden interno ${ }^{10}$.

En primer lugar, estos cuatro principios son vinculantes para las instituciones de la Unión, que están obligadas a respetarlos en el marco de las medidas que adopten o de los dictámenes que emitan. De esta manera, todos los actos de legislación secundaria están subordinados a ellos. Sin embargo, este requisito no impide que las instituciones gocen de cierto grado de discreción. Es probable que las disposiciones relativas a la acción de la Unión Europea en materia de medio ambiente - establecidas en el párrafo 3 del artículo 191 del Tratado de Funcionamiento- atenúen, en su caso, el rigor de los principios ${ }^{11}$. Sin embargo, la flexibilidad inherente a los principios no permite que las instituciones los excluyan en forma sistemática.

En segundo lugar, aunque no tengan efecto directo en el derecho interno ${ }^{12}$, los Estados miembros están obligados a respetarlos al aplicar las normas que establecen los principios. Además, aunque estos principios no se enuncien expresamente en la legislación secundaria, aclaran no obstante el alcance de las obligaciones. De conformidad con los principios que se derivan del ordenamiento jurídico de la Unión ${ }^{13}$, el derecho derivado debe aplicarse según los principios proclamados en el artículo 191 del Tratado de Funcionamiento. El Tribunal de Justicia de la Comunidad Europea interpreta las obligaciones del derecho secundario a la luz de estos principios.

10 Nicolas de Sadeleer, Environmental Principles: from Political Slogans to Legal Rules, $2 .^{\mathrm{a}}$ ed. (Oxford: Oxford University Press, 2020).

11 A modo de ejemplo, la Comisión acepta la validez de ciertas ayudas estatales en materia de medio ambiente que son, a primera vista, contrarias al principio de que quien contamina paga, debido a que las regiones en las que se encuentran las empresas beneficiarias de las ayudas están económicamente atrasadas.

12 Conclusiones del abogado general Lens en el marco de la Corte, $\mathrm{I}_{4}$ de julio de r994, Peralta, C-379/92, Rec., p. I-3453, punto $5^{8 .}$

13 Corte de Justica, Io de abril de 1984 , von Colson con Kamann, C-I $4 / 83$, Rec., p. I891, punto 26; Corte de Justicia, I3 de noviembre de I990, Marleasing, C-Io6/89, Rec., p. I-_-125, punto 8. 
Sin embargo, la situación no es tan idílica. Los principios enunciados se formulan de manera general sin definir sus condiciones y modalidades de aplicación. El hecho de que los autores del Tratado no les dieran prioridad significa que deben ser sopesados entre $\mathrm{si}^{14}$. Estos principios pueden ser confrontados con otros principios generales del derecho de la Unión, como el de la seguridad jurídica ${ }^{15}$. Por último, muchos principios recientes (de no regresión, de sustitución, de participación, de compensación ecológica, etcétera) no están contemplados en el derecho primario.

\section{UN DERECHO FUNDAMENTAL A LA PROTECCIÓN DEL MEDIO AMBIENTE EN EL ORDENAMIENTO JURÍDICO DE LA UNIÓN EUROPEA}

Fuertemente marcado en el origen por el objetivo de la integración económica, el ordenamiento jurídico de la Unión Europea se basa en una serie de libertades fundamentales (libertad de circulación, libre competencia), que pueden entrar en conflicto con los derechos fundamentales (derecho a la libertad de expresión ${ }^{16}$, derecho a la protección judicial y otras).

A diferencia de las empresas, que pueden invocar ante sus tribunales nacionales los derechos económicos en el Tribunal de Funcionamiento, los particulares no pueden invocar las disposiciones del derecho primario dedicadas a la protección del medio ambiente. A falta de normas de derecho primario directamente aplicables, corresponde al legislador organizar la protección de los particulares, si es necesario mediante disposiciones claras y precisas que sean directamente aplicables.

Dicho esto, no se puede ignorar que, por una parte, el Convenio Europeo de Derechos Humanos - algunas de cuyas disposiciones se refieren en forma indirecta a cuestiones ambientales $^{17}$ - es una fuente privilegiada para determinar los principios generales del derecho de la Unión Europea. En efecto, desde hace veinte años, se interpreta que el

14 Corte de Justicia de la Unión Europea, I 4 de julio de I998, Safety Hi-Tech, C-321/95, Rec., p.

I-4355, punto 35 .

15 Corte de Justicia, I8 de junio de i998, Gedeputeerde Staten con Noord-Holland, C-8I/96, Rec., p. I-3923, puntos 23-24; Corte de Justicia, 23 de marzo de 2006, Commission con Autriche, C-209/o, Rec., p. I-2755, punto 57 .

16 Corte de Justicia de la Unión Europea, I2 de junio de 2003, Schmidberger, C-II2/02, Rec., p. I-5659.

17 Fallo del 20 de diciembre de 20 I9 del Hoge Raad de los Países Bajos, Urgenda, ECLI:NL:RBDHA:20I5:7145. Este fallo es de suma importancia. El cambio climático es, en última instancia, una amenaza considerable para el disfrute de los derechos fundamentales (artículos 2 y 8 de la Convención) y para la propia habitabilidad de regiones enteras del planeta. 
Convenio Europeo de Derechos Humanos impone a los Estados obligaciones relativas a la protección del medio ambiente. El derecho a la vida y el derecho al respeto de la vida privada y familiar han servido de puerta de entrada. Revestida con las características de un Estado de derecho, la Unión Europea reconoce estos derechos fundamentales "como principios generales" (artículo 6, párrs. 1 y 3). Según la jurisprudencia del Tribunal de Justicia, el respeto de los derechos humanos es una condición para la legalidad de los actos de la Unión Europea (Dictamen 2/94, punto 34). Las medidas incompatibles con el respeto de los derechos humanos no pueden permitirse en la Unión Europea ${ }^{18}$.

Dicho esto, el recurso exclusivo a los derechos humanos, a través del CEDH, para proteger el medio ambiente parece insatisfactorio. La prueba de su violación requiere la demostración de que a menudo es mucho más grave (lesiones graves, intoxicaciones, trastornos sicológicos profundos) que la que podría invocarse en un litigio administrativo o civil (superación de un umbral, falta de autorización, etcétera) ${ }^{19}$.

Si bien el Convenio Europeo de Derechos Humanos parece, pues, de poca ayuda, merece nuestra atención la obligación positiva impuesta a las instituciones y, por ende, a los Estados miembros, de procurar un alto nivel de protección del medio ambiente, en virtud del artículo 37 de la Carta de Derechos Fundamentales.

El artículo 37 prescribe la integración de la protección del medio ambiente en todas las políticas de la Unión, agregando así diversas disposiciones de derecho primario preexistentes sobre la protección del medio ambiente - que es uno de los objetivos esenciales de la Unión en virtud del artículo 3.3 del Tratado-y declarando su nivel de ambición (un alto nivel de protección y mejora de su calidad).

Aunque en esta etapa el Tribunal de Justicia ha emitido pocos fallos sobre el alcance del artículo 37, esta disposición aparece con mayor fuerza en las conclusiones de varios abogados generales en numerosos $\operatorname{casos}^{20}$.

18 Corte de Justicia, I2 de junio de 2003 , Schmidberger, C-Ir2/oo, [2003] ECR I-5659, párrafo 73; Corte de Justicia, 3 de septiembre de 2008, Kadi, C-402/05 P y C-415/05 P, [2008] ECR I-638I, párrafo 284 .

19 Tribunal Europeo de Derechos Humano, López Ostra con España, 9 de diciembre de I994, § 5г; Tribunal Europeo de Derechos Humano, Moreno Gómez con España, I6 de noviembre de 2004; Tribunal Europeo de Derechos Humano, Fadë̈eva con Rusia, 9 de junio de 2005 , § 70.

20 Véanse, en particular, las conclusiones del abogado general Jääskinen en el asunto C-557/I5, Comisión con Malta, del 26 de julio de 20I7, párrafo 44, y en el caso C-664/15, Protect Natur, del I2 de octubre de 20I7, párrafo 68; las conclusiones del abogado general Tanchev en el asunto C-535/ז5, Pinckernelle, del 2r de diciembre de 20I6, punto 73; los hallazgos del difunto abogado general Bot en el caso C-r95/12, Industrie du bois de Vielsam, del 8 de mayo de 2013, párrafo 82; las conclusiones del abogado general Kokott en el caso C-723/13, Craeynest, del 28 de febrero de 20I9, párrafo 53; aff. C-416/ı, Krizan, del I9 de abril de 20I2, punto I85 y C-260/II, David Edwards, del I8 de octubre de 20I2, punto 40; conclusiones de abogado general Colomer en aff. C-87/02, Commission con Italie, 8 de enero de 2004 , punto 36 . 
El cumplimiento del artículo 37 de la Carta determina la validez de la legislación secundaria y, como tal, está sujeta a revisión judicial. Dicho control se ejercerá en las condiciones y límites establecidos en los Tratados ${ }^{21}$, incluidas, en particular, las condiciones y límites resultantes del artículo 191 del Tratado de Funcionamiento ${ }^{22}$.

El artículo 37 de la Carta también se inscribe como un elemento de interpretación de la legislación secundaria y, por lo tanto, afecta a las políticas nacionales en materia de medio ambiente.

Ayuda a impulsar un movimiento fundamental para aumentar la importancia que debe darse al medio ambiente como interés protegido, que no debe permitirse que caiga presa de intereses socioeconómicos conflictivos desde el principio.

\section{CONCLUSIONES}

La incorporación del desarrollo sustentable, la obligación de integración, la consolidación de las bases jurídicas de una política específicamente dedicada a este objeto, la afirmación de los principios, son todos elementos a favor del reconocimiento de un valor fundamental del ordenamiento jurídico de la Unión Europea. Además, solo gradualmente, bajo la presión del principio de integración, las preocupaciones ambientales están encontrando su camino en otras áreas de la legislación de la Unión.

Como objetivo de interés de la Unión Europea, la protección del medio ambiente como nueva faceta del interés general permite a las autoridades públicas desplegar medidas policiales que pueden restringir el alcance de los derechos económicos o incluso fundamentales. El alcance de las obligaciones en virtud de la legislación secundaria debe interpretarse a la luz de este imperativo.

Este reconocimiento no debería en ningún caso crear ilusiones. En primer lugar, las disposiciones mencionadas incluyen conceptos emblemáticos (desarrollo sustentable, integración, alto nivel de protección) pero son muy ambiguas. Además, la cuestión aquí es menos de priorización que de conciliación de intereses. No es seguro que el medio ambiente sea el ganador.

Sin embargo, lo más importante es que estas mejoras no han puesto en tela de juicio la preponderancia de los intereses económicos garantizados por los tratados fundacionales.

21 Corte de Justicia de la Unión Europea, 2r de diciembre de 20I6, Italia Nostra Onlus con Commune di Venezia, aff. C-444/ı5, ECLI:EU:C:2016:978, puntos 6r a 64.

22 Corte de Justicia de la Unión Europea, I3 de marzo de 20r9, Pologne c. Parlement et Conseil, aff. C-I28/I7, puntos I29 a I3I. 
En efecto, la integración europea está sobre todo enraizada en la integración económica, mientras que las cuestiones ambientales — como las de la salud - solo se han abordado hasta ahora de manera accesoria. Esta diferencia de trato es evidente cuando se trata de la situación de los derechos económicos. Por parte del Tribunal, los artículos 34 y 35 del Tratado de Funcionamiento prohíben las medidas de efecto equivalente a las restricciones cuantitativas al comercio ${ }^{23}$, el artículo 101 del Tratado de Funcionamiento tiene por efecto hacer ilegales los acuerdos contrarios a la competencia entre empresas, mientras que el artículo 107 prohíbe en principio las ayudas estatales ${ }^{24}$. En el lado del jardín, los artículos 191 a 193, en torno a los cuales se estructura la política ambiental, son disposiciones habilitantes sin efecto directo. Además, no prohíben en modo alguno los riesgos ambientales significativos.

Es cierto que se objetará que el Tratado de Funcionamiento obliga a las instituciones a alcanzar un alto nivel de protección del medio ambiente y a integrar esas preocupaciones en otras políticas, pero esas obligaciones deben traducirse en términos concretos en cuanto a las medidas de armonización que deben adoptarse en todas las esferas de intervención. El Tratado solo define un marco; en efecto, su aplicación depende de la buena voluntad en primer lugar de las instituciones y en segundo lugar de las autoridades nacionales.

Por último, no se han hecho progresos significativos en la legislación de la Unión Europea en favor del acceso a la justicia para los ciudadanos y las organizaciones ambientales o en el establecimiento de un derecho a la protección del medio ambiente.

23 Peter Olivier, "Some Further Reflections on the Scope of Articles 28-3० (ex $3^{\circ-36)}$ EC", Common Market Law Review 36, n. ${ }_{4}^{4}$ (I999): 80,-806.

24 Nicolas de Sadeleer, EU Environmental Law and the Internal Market (Oxford: Oxford University Press, 20I/). 


\section{BIBLIOGRAFÍA}

- Alves, Carlos-Manuel. "La protection intégrée de l'environnement en droit de l'UE”. Tesis de la Universidad de Montesquieu-Burdeos IV, 2002.

- D'Hondt, Nele. Integration of Environmental Protection into other European Community Policies: Legal Theory and Practice. Groninga: Europa Law Publishing, 2003.

- De Sadeleer, Nicolas. Commentaire Mégret Environnement et marché intérieur. Bruselas: Éditions de l'Université libre de Bruxelles, 2010.

- - EU Environmental Law and the Internal Market. Oxford: Oxford University Press, 2014.

- Environmental Principles: from Political Slogans to Legal Rules. 2. ${ }^{\mathrm{a}}$ edición. Oxford: Oxford University Press, 2020.

- Olivier, Peter. "Some Further Reflections on the Scope of Articles 28-30 (ex 30-36) EC”. Common Market Law Review 36, n. ${ }^{\circ} 4$ (1999): 783-806.

- Wasmeier, Martin. "The Integration of Environmental Protection as General Rule for Interpretating Law". Common Market Law Review 38, n. ${ }^{\circ} 1$ (2001): 159-177. 